\title{
The new method, the old problem - role of contrast-enhanced spectral mammography in the diagnosis of breast cancer among Polish women
}

\author{
Katarzyna Steinhof-Radwańska ${ }^{1 A, B, C, D, E, F, G}$, Anna Grażyńska ${ }^{2 B, C, E, F G}$, Anna Barczyk-Gutkowska ${ }^{1 B, D, E, F, G}$, Maciej Kajor ${ }^{3 B, D, E}$, \\ Piotr Powązka ${ }^{4 B, D, F}$, Andrzej Lorek ${ }^{5 B, F, G}$, Ewa Szlachta-Świątkowska ${ }^{5 B, F}$, Irmina Morawska ${ }^{2 B, C, E}$, Karolina Okas ${ }^{2 B, C, E}$, \\ Zuzanna Lelek ${ }^{2 B, C E}$, Magdalena Bielińska ${ }^{2 \mathrm{E}}$, Iwona Gisterek ${ }^{6 \mathrm{E}, \mathrm{F}}$, Beata Casañas ${ }^{\mathrm{TE}}$, Joanna Pilch-Kowalczyk ${ }^{1 \mathrm{E}, \mathrm{F}}$
}

'Department of Radiology and Nuclear Medicine, Medical University of Silesia in Katowice, Poland

2Students' Scientific Society, Department of Radiology and Nuclear Medicine, Medical University of Silesia in Katowice, Poland

${ }^{3}$ Department of Pathomorphology and Molecular Diagnostics, Medical University of Silesia in Katowice, Poland

${ }^{4}$ Department of Imaging Diagnostics and Interventional Radiology, University Clinical Center in Katowice, Poland

${ }^{5}$ Department of Oncological Surgery, Medical University of Silesia in Katowice, Poland

${ }^{6}$ Department of Oncology and Radiotherapy, Medical University of Silesia in Zabrze, Poland

${ }^{7}$ Division of Infectious Diseases and International Health, Department of Medicine, Morsani, College of Medicine, University of South Florida, USA

\section{Abstract}

Purpose: The aim of the study was to evaluate spectral mammography (CESM) in diagnosing breast cancer, which is based on sensitivity, specificity, positive predictive value (PPV), and negative predictive value (NPV).

Material and methods: The study included a group of 547 women who underwent spectral mammography and histopathological verification of the lesion, previously seen in mammography and/or ultrasound. In the group of 547 women, 593 focal lesions were diagnosed. All CESM examinations were carried-out with a digital mammography device dedicated to performing dual-energy CESM acquisitions. An intravenous injection of $1.5 \mathrm{ml} / \mathrm{kg}$ of body mass of non-ionic contrast agent was performed.

Results: The analysis includes 593 breast lesions, in this group cancer was detected in 327 (55.14\%) lesions, and in $256(43.17 \%)$ cases benign lesions were confirmed by histopathological examination and at least 12 months of observation. The method shows differentiation of benign and malignant lesions in the breast: sensitivity of $97.86 \%$, specificity of $59.4 \%$, PPV $-74.76 \%$, NPV $-95.76 \%$.

Conclusions: Spectral mammography could be an ideal method to detect breast cancer. Thanks to the high NPV (95.76\%), it facilitates the exclusion of cancer in situations where pathological contrast enhancement is not observed. The unsatisfactory specificity of the study $(59.4 \%)$ would not make it safe to avoid a core needle biopsy of lesions that undergo contrast enhancement.

Key words: breast cancer diagnostics, spectral mammography (CESM), mammography (MG).

\section{Introduction}

Breast cancer is the most frequently diagnosed malignancy, with a frequency of $22.8 \%$ of all new cancer incident rates in Poland [1]. The most frequent occurrence is found among women in ages 50-69 years, whereas below the age of 40 years it is not a frequent disease. However, recent observations indicate an increasing number of diagnosed cases of breast cancer being reported in pre-menopausal women [2]. According to the National Cancer Registry

Correspondence address:

Katarzyna Steinhof-Radwańska, Department of Radiology and Nuclear Medicine, Medical University of Silesia, 35 Ceglana St., 40-514 Katowice, Poland,

e-mail: kasia.steinhof@gmail.com

Authors' contribution:

A Study design · B Data collection · C Statistical analysis · D Data interpretation · E Manuscript preparation · F Literature search · G Funds collection 
(Krajowy Rejestr Nowotworów) the incident rate in adult women of pre-menopausal age (20-49 years) has indeed shown an increasing trend, and the incidence rate has almost doubled in the last three decades [1].

The basic method of breast cancer detection is X-ray mammography, which is widely available, cheap, and repeatable; however, it has two important limitations. Firstly, the ionising radiation and secondly cancer imaging in thick glandular breasts, which can lead to a decrease in sensitivity [3]. Magnetic resonance imaging (MRI) mammography, extended with diffusion imaging (DWI/ADC) is a test with a high level of sensitivity and specificity (over 85\%) [4-6]; however, its limitations are worth noting: it requires an intravenous contrast agent, an uncomfortable position to perform the procedure (prone position), and an acquisition time of 20-30 minutes. Additionally, in breast MRI microcalcifications are not visible.

Other approaches, such as optical methods, with tomography laser mammography among them, is associated with a relatively high number of undiagnosed cancers and an even higher number of false positive results, thus offering no advantage over existing methods [7]. Considering tomosynthesis, the main benefit is a higher cancer detection rate. Unfortunately, this method has its disadvantages, such as high radiation dose and limited capability in detecting microcalcifications [8].

Contrast-enhanced spectral mammography (CESM) is a new technique intensively developed in the last few years and accepted by the FDA for clinical use in the U.S. in 2011. This method, like MRI, is based on imaging of tumour neoangiogenesis by use of contrast agent (chelated iodine-based X-ray contrast agent) $[9,10]$. The aim of the study was to evaluate CESM in diagnosing breast cancer, based on sensitivity, specificity, positive predictive value (PPV), and negative predictive value (NPV).

\section{Material and methods}

The retrospective study on 547 patients aged 21 to 89 years (median age 56, interquartile range 46-65 years) was conducted from July 2014 to July 2019. Every patient had ultrasonography (US) and/or conventional mammography (MMG) followed by spectral mammography. Detected lesions were classified using the Breast Imaging-Reporting and Data System (BIRADS). The BIRADS score was created by the American College of Radiology (ACR) in order to standardise radiological descriptions of mammography, US, and MRI studies. According to this score, 0 stands for lesion of unknown character, which needs additional imagining evaluation, 1 is regular breast tissue, 2 represents benign lesions, 3 - lesions most probably benign, 4 - lesions suspicious of malignancy, 5 - lesion highly suggestive of malignancy, and 6 - known biopsy-proven malignancy. Every lesion that scored BIRADS 0 and 4-6 in USG or MMG studies was verified with core needle biopsy. In the group of patients staged BIRADS 4-6 in whom additional lesions were detected and initially classified as BIRADS 2 or 3, those lesions were also verified with core needle biopsy.

Fresh specimens received by core biopsy were sent to the Pathology Department and underwent formalin fixation followed by paraffin embedding. Tumours were diagnosed histologically according to the World Health Organisation classification.

Every woman in this group have conventional mammography done. Additionally, spectral mammography and core needle biopsy were performed among patients who came to our centre with a mammography description from elsewhere stating BIRADS 2 or 3 and the surgeon who performed physical examination had doubts.

Only patients who gave informed consent for further examinations were qualified to spectral mammography studies. Before qualifying to CESM, all patients completed a questionnaire, based on which patients with the possibility of pregnancy were eliminated from the study. Creatinine and GFR (glomerular filtration rate) levels were assessed in each patient.

Exclusion criteria were an eGFR less than $30 \mathrm{ml} / \mathrm{min}$ and an allergic reaction to iodinated or gadolinium-based contrast agents. Patients with BCRA1-BCRA2 mutation were also excluded from studies; this group should be examined via MRI because of lack of radiation.

The study was conducted according to the principles of the Declaration of Helsinki, all participants provided informed written consent, and the study received approval from the Ethical Committee of the Medical University of Silesia in Katowice, Poland.

All CESM examinations were carried-out with a digital mammography device dedicated to the performance of dual-energy CESM acquisitions (SenoBright, GE Healthcare). An intravenous injection of $1.5 \mathrm{ml} / \mathrm{kg}$ of body mass of non-ionic contrast agent was performed using a power injector at a rate of $3 \mathrm{ml} / \mathrm{s}$ with a bolus chaser of $30 \mathrm{ml}$ of saline. In CESM mode, the device automatically performed a pair of exposures (low- and high-energy) in each view. Specific image processing of low-energy and highenergy exposures was done to obtain subtraction images to highlight contrast enhancement and suppress structure noise due to fibroglandular breast tissue [11]. The total study time was usually about 10 minutes. Patients after the examination were observed for the appearance of adverse reactions.

\section{Statistical analysis}

The statistical analysis included the comparison of baseline clinical characteristics, the results of the spectral mammography analysis, and the histopathological results of each lesion. The analysed variables are expressed as numbers and percentages. The normality of the distribution was assessed using the Shapiro-Wilk test and the continuous variables were summarised using an arithme- 
tic mean with standard deviation for data following normal distribution or a median with a quartile 1 and 3 for data demonstrating non-normal distribution. Categorical variables were presented using frequency tables for both absolute numbers and percentages. As in no continuous variable, the distribution was normal, the Mann-Whitney $U$ test was used for the comparisons between the independent values. The differences between the qualitative parameters were assessed utilising the $\chi^{2}$ test with Yates's correction, and Fisher's test if the analysed populations were insufficiently represented. Sensitivity, specificity, PPV, and NPV of the CESM method were calculated according to the following formulas:

- sensitivity $=[\mathrm{TP} /(\mathrm{TP}+\mathrm{FN})] \times 100 \%$,

- $\quad$ specificity $=[\mathrm{TN} /(\mathrm{TN}+\mathrm{FP})] \times 100 \%$,

- $\mathrm{PPV}=[\mathrm{TP} /(\mathrm{TP}+\mathrm{FP})] \times 100 \%$,

- $\mathrm{NPV}=[\mathrm{TN} /(\mathrm{TN}+\mathrm{FN})] \times 100 \%$.

A two-sided $p$-value $\leq 0.05$ was considered significant. For sensitivity and specificity analysis, the area under the curve (AUC) for binary variables of tumour enhancement in CESM and tumour malignancy in histopathology was performed. STATISTICA 10 (StatSoft Inc., Tulsa, Oklahoma) software was used for all calculations.

\section{Results}

The analysis includes 593 breast lesions diagnosed in 547 women. In the studied group cancer was detected in 327 (55.14\%) lesions, and in 256 (43.17\%) cases benign lesions were confirmed by histopathological examination and at least 12 months of observation. In 10 lesions (1.69\%) lobular carcinoma in situ (LCIS) was diagnosed, which in histopathological examination was deemed to be a benign lesion, but for the purpose of this study LCIS was classified as indirect malignancy. The most common malignant lesion was NST (non-specific type) cancer (68.5\%). In the group of benign lesions, the most common diagnosis was fibrotic sclerosis $(57.42 \%)$. The frequency of particular lesions is presented in Table 1.

In the CESM study, the changes took the following variants: 13 (2.19\%) microcalcifications, 107 (18.03\%) tumours, 221 (37.27\%) microcalcifications and asymmetry, 159 (26.81\%) microcalcifications and tumour, and 91 (15.35\%) asymmetry and tumour. As much as $97.9 \%$ of malignant lesions were enhanced by contrast - among benign and intermediate lesions only $40 \%$ of each. From all of the lesions enhanced by contrast $74.77 \%$ were malignant, $24.30 \%$ were benign, and $0.93 \%$ were of intermediate malignancy. In most cases of lesions without contrast enhancement the character was benign (92.12\%), only $4.24 \%$ of non-enhanced lesions were malignant. The $\chi^{2}$ test showed a statistically significant difference between contrast enhancement and histopathological result (Table 2). The size of the changes ranged from $1 \mathrm{~mm}$ to $150 \mathrm{~mm}$ (median: $19 \mathrm{~mm}$, interquartile range 12-30). Contrast enhancement depends also on the size of the lesion. The median
Table 1. Pathology summary for included malignant, benign, and possibly benign lesions

\begin{tabular}{|l|c|}
\hline Lesion types & $n(\%)$ \\
\hline NST & $224(37.77)$ \\
\hline DCIS & $55(9.27)$ \\
\hline Infiltrating lobular carcinoma & $37(6.23)$ \\
\hline Metaplastic carcinoma & $4(0.67)$ \\
\hline Invasive mucinous carcinoma & $5(0.84)$ \\
\hline Invasive medullar carcinoma & $2(0.34)$ \\
\hline Fibroadenoma & $81(13.66)$ \\
\hline Fibrotic sclerosis & $147(24.8)$ \\
\hline Papilloma & $16(2.70)$ \\
\hline LCIS & $10(1.70)$ \\
\hline Other & $12(2.02)$ \\
\hline
\end{tabular}

NST - non-specific type cancer, DCIS - pre-invasive ductal carcinoma, LCIS - lobular carcinoma in situ, 0ther - atheroma, microglandular lobular hyperplasia, focal apocrine metaplasia, usual ductal hyperplasia, cyst, phyllodes tumour

Table 2. Presentation of all enhanced (CESM [+]) or non-enhanced (CESM [-]) lesions depending on the lesion malignancy

\begin{tabular}{|c|c|c|c|}
\hline $\begin{array}{l}\text { Lesion } \\
\text { characteristics }\end{array}$ & $\begin{array}{c}\operatorname{CESM}(+), \\
n(\%)\end{array}$ & $\begin{array}{c}\operatorname{CESM}(-), \\
n(\%)\end{array}$ & $P \chi^{2}$ \\
\hline Malignant & $320 / 428(74.77)$ & $7 / 165$ (4.24) & \multirow[t]{3}{*}{$<0.0001$} \\
\hline Benign & $104 / 428(24.30)$ & $152 / 165(92.12)$ & \\
\hline Possibly benign & $4 / 428(0.93)$ & $6 / 165(3.64)$ & \\
\hline
\end{tabular}

CESM - contrast-enhanced spectral mammography

size for enhanced lesions was $20.0 \mathrm{~mm}$ (12.0-30.0), and for those without enhancement it was $13.0 \mathrm{~mm}(8.0-23.0)$ (Mann-Whitney's $U$ test, $p<0.0001$ ).

Table 3 shows the correlation between contrast enhancement and BIRADS score with divisions according to histopathological results.

Table 4 presents how particular lesion enhanced conforming to histopathological result.

\section{Sensitivity, specificity, positive predictive value, negative predictive value, and ROC}

In 428 (72.2\%) lesions changes of increased vascularisation were detected (CESM [+]), while in the remaining 165 (27.8\%) lesions the CESM result was negative (CESM [-]). The CESM (+) result was truly positive in $320(53.96 \%)$ cases (Figure 1) and false positive in 158 (26.64\%) cases. The CESM (-) result was truly negative (HP-) in 108 (18.21\%) cases (10 LCIS low-grade lesions were specified in the histopathology for sensitivity, specificity, PPV, and NPV and were classified as benign) and false negative in 7 (1.18\%) cases (5 DCIS and 2 NST). Taking the CESM (+) result as the criterion of malignancy, the method 
Table 3. Correlation between BIRADS classification and contrast enhancement. Presentation of histopathology results depending on BIRADS

\begin{tabular}{|l|c|c|c|c|c|c|c|}
\hline $\begin{array}{l}\text { BIRADSI } \\
\text { Enhancement }\end{array}$ & $\begin{array}{c}0 \\
(n=2)\end{array}$ & $\begin{array}{c}2 \\
(n=13)\end{array}$ & $\begin{array}{c}3 \\
(n=107)\end{array}$ & $\begin{array}{c}4 \\
(n=221)\end{array}$ & $\begin{array}{c}5 \\
(n=159)\end{array}$ & $\begin{array}{c}6 \\
(n=91)\end{array}$ & \begin{tabular}{c}
$P \chi^{2}$ \\
\hline CESM $(+)(n=428)$
\end{tabular} \\
\hline CESM $(-)(n=165)$ & $50 \%(1 / 2)$ & $46.1 \%(6 / 13)$ & $51.4 \%(55 / 107)$ & $54.7 \%(121 / 221)$ & $98.1 \%(156 / 159)$ & $97.8 \%(89 / 91)$ & $<0.001$ \\
\hline Histopathology type & $0(n=2)$ & $2(n=13)$ & $3(n=107)$ & $4(n=221)$ & $5(n=159)$ & $6(n=91)$ \\
\hline Fibrotic sclerosis & $0(0 / 2)$ & $30.8 \%(4 / 13)$ & $45.8 \%(49 / 107)$ & $41.2 \%(91 / 221)$ & $1.4 \%(2 / 159)$ & $2.19 \%(2 / 91)$ & \\
\hline Fibroadenoma & $0(0 / 2)$ & $46.1 \%(6 / 13)$ & $38.3 \%(41 / 107)$ & $15.4 \%(34 / 221)$ & $0(0 / 159)$ & $0(0 / 91)$ \\
\hline NST & $0(0 / 2)$ & $7.7 \%(1 / 13)$ & $2.8 \%(3 / 107)$ & $14.9 \%(33 / 221)$ & $76.7 \%(122 / 159)$ & $72.52 \%(66 / 91)$ \\
\hline DCIS & $100 \%(2 / 2)$ & $0(0 / 13)$ & $1.8 \%(2 / 107)$ & $10.9 \%(24 / 221)$ & $6.3 \%(10 / 159)$ & $17.58 \%(16 / 91)$ \\
\hline Papilloma & $0(0 / 2)$ & $0(0 / 13)$ & $4.7 \%(5 / 107)$ & $4.5 \%(10 / 221)$ & $0.6 \%(1 / 159)$ & $0(0 / 91)$ \\
\hline ILC & $0(0 / 2)$ & $7.7 \%(1 / 13)$ & $0.9 \%(1 / 107)$ & $4.1 \%(9 / 221)$ & $13.8 \%(22 / 159)$ & $4.39 \%(4 / 91)$ \\
\hline LCIS & $0(0 / 2)$ & $0(0 / 13)$ & $0.9 \%(1 / 107)$ & $3.2 \%(7 / 221)$ & $0(0 / 159)$ & $0(0 / 91)$ \\
\hline Other & $0(0 / 2)$ & $7.7 \%(1 / 13)$ & $3.7 \%(4 / 107)$ & $3.6 \%(8 / 221)$ & $1.4 \%(2 / 159)$ & $3.29 \%(3 / 91)$ & \\
\hline
\end{tabular}

NST - non-specific type cancer, DCIS - pre-invasive ductal carcinoma, ILC - infiltrating lobular carcinoma, LCIS - lobular carcinoma in situ, 0ther - focal apocrine metaplasia, invasive mucinous carcinoma, atheroma, usual ductal hyperplasia, metaplastic carcinoma, microglandular hyperplasia, invasive medullar carcinoma, cyst, phyllodes tumour

Table 4. Enhancement of individual lesions depending on the histopathological results

\begin{tabular}{|l|c|c|c|}
\hline Lesion & $\begin{array}{c}\text { Quantity } \\
\text { (\% of total) }\end{array}$ & CESM (+) & CESM (-) \\
\hline Fibrotic sclerosis & $24.8 \%(147 / 593)$ & $15.7 \%(23 / 147)$ & $84.3 \%(124 / 147)$ \\
\hline Fibroadenoma & $13.7 \%(81 / 593)$ & $77.8 \%(63 / 81)$ & $22.2 \%(18 / 81)$ \\
\hline NST & $37.8 \%(224 / 593)$ & $99.1 \%(222 / 224)$ & $0.9 \%(2 / 224)$ \\
\hline DCIS & $9.3 \%(55 / 593)$ & $90.9 \%(50 / 55)$ & $9.1 \%(5 / 55)$ \\
\hline Papilloma & $2.7 \%(16 / 593)$ & $93.8 \%(15 / 16)$ & $6.2 \%(1 / 16)$ \\
\hline ILC & $6.2 \%(37 / 593)$ & $100.0 \%(37 / 37)$ & $0 \%(0 / 37)$ \\
\hline LCIS & $1.3 \%(8 / 593)$ & $25 \%(2 / 8)$ & $75 \%(6 / 8)$ \\
\hline 0ther & $4.2 \%(25 / 593)$ & $64.0 \%(16 / 25)$ & $36.0 \%(9 / 25)$ \\
\hline
\end{tabular}

NST - non-specific type cancer, DCIS - pre-invasive ductal carcinoma, ILC - infiltrating lobula carcinoma, LCIS - lobular carcinoma in situ, 0ther - focal apocrine metaplasia, invasive mucinous carcinoma, atheroma, usual ductal hyperplasia, metaplastic carcinoma, microglandular hyperplasia, invasive medullar carcinoma, cyst, phyllodes tumour

shows differentiation of benign and malignant lesions in the breast: sensitivity of $97.86 \%$, specificity of $59.4 \%$; PPV 74.76\%; NPV 95.76\% (Figure 2). The area under the curve (AUC) for the binary variables (CESM enhancement and malignancy in histopathology) was 0.654 (Figure 3).

In this study, we also decided to check the effectiveness of CESM in preinvasive cancers. For DCIS the CESM $(+)$ result was truly positive in $50(90.91 \%)$ cases and false negative in $5(9.09 \%)$.

\section{Discussion}

Our study presents the largest population of women diagnosed via CESM in Poland and one of the largest populations of women who had this imagining done in the world. In the group of 593 lesions included in the analysis, there were 327 (55.14\%) cancers, 256 (43.17\%) benign lesions, and $10(1.69 \%)$ lesions determined by pathologists on the basis of core needle biopsy and subsequent neoplastic tumourectomy as probably mild. A positive CESM result was obtained more frequently in cases of breast cancer (97.86\%) than benign lesions (40.63\%). The significantly more frequent CESM (+) result in the group of cancers than found in mild lesions, can be explained by the presence of a dense pathological bed in malignant tumours. Assuming the CESM (+) result as a diagnostic criterion for malignant lesions in its own material, the sensitivity of this method was determined at $97.86 \%$ and specificity at $59.4 \%$. Similar results were obtained by Łuczyńska, who presented the sensitivity of CESM at $100 \%$ [12], as well as Fallenber [13] and Loobes [14].

In our material, 259 of 261 invasive carcinomas showed pathological contrast enhancement, as well as 50 of 55 DCIS (pre-invasive ductal carcinomas). Cancers without pathological enhancement were dominated by necrosis, which could affect the results. Enhancement in the case of DCIS can be explained by the increased density of vessels in the stroma between the infiltrated ducts, because DCIS has no ability to induce angiogenesis, unlike invasive cancers [15]. Only five cancers (DCIS low grade) showed no pathological vascularisation. These lesions represented microcalcifications, without evident tumour mass. Problems with the differential diagnosis of microcalcifications in breast cancers were described by Houben [16].

The sensitivity of the CESM examination is comparable to magnetic resonance, which means that these are the most sensitive methods used in breast diseases. Unfortunately, the specificity of CESM is clearly lower than the sensitivity. In our material the specificity of the method was obtained at the level of $59.4 \%$. More than $1 / 3$ of benign lesions showed abnormal contrast enhancement.

The achieved specificity of $59.4 \%$ is comparable to the results of Łuczyńska [12], where as many as $60 \%$ of benign 


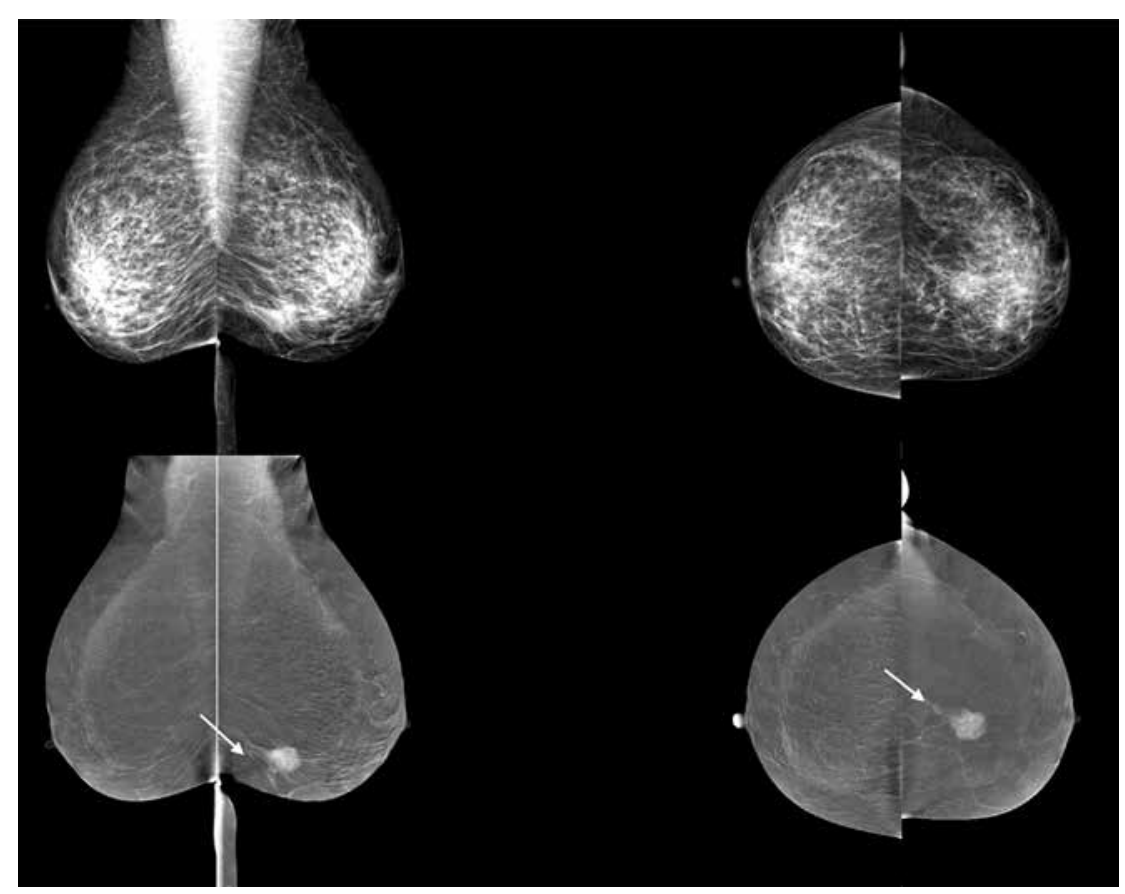

Figure 1. True positive result (TP). Left MLO (mediolateral view) and CC (craniocaudal) contrast-enhanced spectral mammography reveals avid enhancement of the mass (arrow) with non-mass enhancement extending posteriorly (arrowhead) in lower internal quadrant of the breast. Left MLO (mediolateral view) and CC (craniocaudal) low-energy image shows an irregular mass with indistinct margin in lower internal quadrant of the breast BIRADS $4 C$

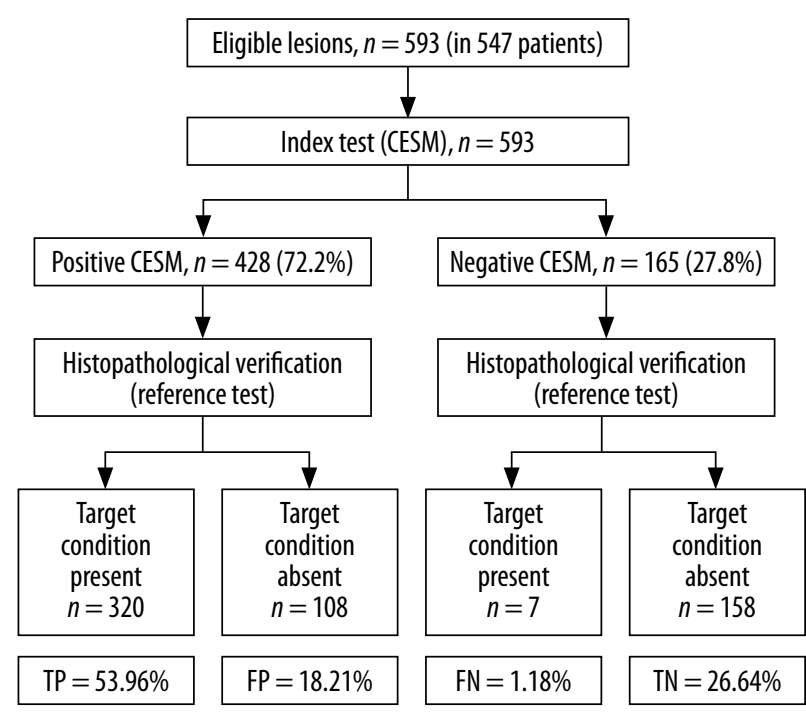

Figure 2. Comparison of lesions clinical characteristics and the results of the spectral mammography analysis

lesions showed features of contrast enhancement. Similar values were obtained in the meta-analysis [17], which included 994 lesions, yielding a specificity of 0.58 .

The specificity of CESM is clearly lower than the specificity of magnetic resonance imaging. Of the benign lesions that were enhanced, fibroadenomas and papillomas predominate. The degree of vascularisation of fibroadenomas is very different and depends on the mutual proportions of fibrous and cellular elements [18]; the more the nodule is hypercellular, the greater its degree of vascularity. In our material $77.8 \%$ of fibroadenomas showed contrast enhancement. Papillomas have a vascular peduncle and perhaps it may be responsible for the contrast enhancement within them - in our material as

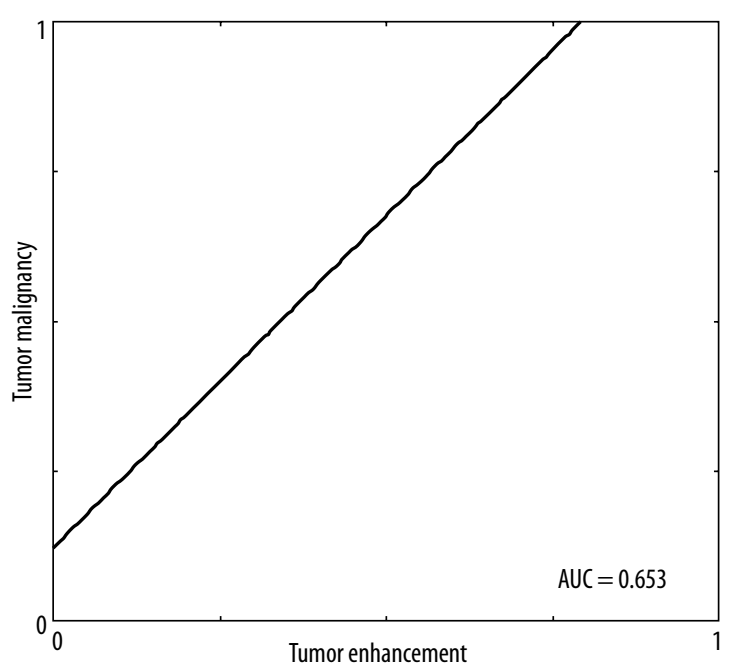

Figure 3. Tumor malignancy verified in histopathology vs. enhancement in contrast-enhanced spectral mammography

much as $91.7 \%$ of papillomas were contrasting. However, lesions visualised by magnetic resonance display high signal intensity on T2-weighted images. Therefore, additional dynamic contrast-enhanced MR (CEM) is necessary to confirm the diagnosis [19]. However, magnetic resonance also at the beginning of its inception struggled with the problem of low specificity, and only the assessment of morphological and dynamic features combined contributed to an increase in specificity to $74-96 \%$, on average around $80 \%$ [20-22]. Unfortunately, the possible assessment of the curve of the contrast enhancement dynamics in CESM (analogous to the MR) seems unlikely due to the presence of ionising radiation in CESM, which does not allow the increase of the number of projections 
over those that are normally performed. The high NPV (negative predictive value) of $95.76 \%$ allows a very high probability of excluding cancer in situations where pathological contrast enhancement is not observed.

In our study BIRADS classification was based on classic diagnostic imaging - US and mammography. There were 120 lesions classified as BIRADS 2 or 3, of which $61(50.82 \%)$ were enhanced and six (5\%) were invasive breast cancer. Therefore, after analysis in a routine work, we adopted the principle that if the changes in BIRADS 2 and 3 stages were enhanced, then its classification would increase to BIRADS 4 and could be qualified for core needle biopsy.

\section{Conclusions}

Spectral mammography (CESM) due to its very high sensitivity may be the preferred method used to detect breast cancer. Due to the high NPV (95.76\%) CESM allows the possibility of excluding cancer in situations where pathological contrast enhancement is not observed, and this may decrease the need for core needle biopsies in cases that are classified in classic imaging (MMG, USG) for biopsy.

\section{Conflict of interest}

The authors report no conflict of interest.

\section{References}

1. Didkowska J, Wojciechowska U, Olasek P, Czaderny K. Polish National Cancer Registry [serial online]. Available at: http://onkologia. org.pl/nowotwory-piersi-kobiet/.

2. Wojciechowska U, Czaderny K, Ciuba A, Olasek P, Didkowska J. Nowotwory złośliwe w Polsce w 2016 roku. Cancer in Poland in 2016. Warszawa; 2018. Available at: http://onkologia.org.pl/wp-content/uploads/Nowotwory_2016.pdf.

3. Gilbert FJ, Pinker-Domenig K, Holder J, et al. Diagnosis and staging of breast cancer: when and how to use mammography, tomosynthesis, ultrasound, contrast-enhanced mammography, and magnetic resonance imaging. In: Diseases of the Chest, Breast, Heart and Vessels 2019-2022. p. 155-166, 20 February 2019.

4. Azab EA, Ibrahim ME. Diffusion weighted (DW) MRI role in characterization of breast lesions using absolute and normalized ADC values. Egyptian Journal of Radiology and Nuclear Medicine 2018; 49: 564-570.

5. Yadav P, Chauhan S. Effectivity of combined diffusion-weighted imaging and contrast-enhanced MRI in malignant and benign breast lesions. Pol J Radiol 2018; 83: e82-e93.

6. Yılmaz E, Sarı O, Yılmaz A, et al. Diffusion-weighted imaging for the discrimination of benign and malignant breast masses; utility of ADC and relative ADC. J Belg Soc Radiol 2018; 102: 24.

7. Steinhof-Radwańska K, Bobek-Billewicz B, Gorczewski K, et al. An assessment of computer tomography laser mammography in breast cancer diagnosis. Pol Ann Med 2018; 25: 21-25.

8. Rocha Garcia AM, Mera Fernandez D. Breast tomosynthesis: state of the art. Radiologia 2019; 61: 274-285.

9. Daniaux M, De Zordo T, Santner W, et al. Dual-energy contrastenhanced spectral mammography (CESM). Arch Gynecol Obstet 2015; 292: 739-747.

10. Bhimani C, Matta D, Roth RG, et al. Contrast-enhanced spectral mammography: technique, indications, and clinical applications. Acad Radiol 2017; 24: 84-88.

11. Patel BK, Lobbes MB, Lewin J. Contrast enhanced spectral mammography: a review. Semin Ultrasound CT MRI 2018; 39: 70-79.
12. Łuczyńska E, Niemiec J, Hendrick E, et al. Degree of enhancement on contrast enhanced spectral mammography (CESM) and lesion type on mammography (MG): comparison based on histological results. Med Sci Monit 2016; 22: 3886-3893.

13. Fallenberg EM, Dromain C, Diekmann F, et al. Contrast-enhanced spectral mammography versus MRI: Initial results in the detection of breast cancer and assessment of tumour size. Eur Radiol 2014; 24: 256-264.

14. Lobbes MB, Lalji U, Houwers J, et al. Contrast-enhanced spectral mammography in patients referred from the breast cancer screening programme. Eur Radiol 2014; 24: 1668-1676.

15. Menakuru SR, Brown NJ, Staton CA, Reed MWR. Angiogenesis in pre-malignant conditions. Br J Cancer 2008; 99: 1961-1966.

16. Houben IP, Vanwetswinkel S, Kalia V, et al. Contrast-enhanced spectral mammography in the evaluation of breast suspicious calcifications: diagnostic accuracy and impact on surgical management. Acta Radiol 2019; 60: 1110-1117.

17. Tagliafico AS, Bignotti B, Rossi F, et al. Diagnostic performance of contrast-enhanced spectral mammography: systematic review and meta-analysis. Breast 2016; 28: 13-19.

18. Lo LW, Wong T, Fung EPY, et al. Magnetic resonance imaging of benign breast lesions. Hong Kong J Radiol 2017; 20: 153-164.

19. Klinger K, Bhimani Ch, Shames J, Sevrukov A. Fibroadenoma: from imaging evaluation to treatment. J Am Osteopath Coll Radiol 2019; 8: 17-30.

20. Stusińska M. The value of morphological and dynamic MR mammography image assessment in breast cancer diagnosis. $\mathrm{PhD}$ dissertation. Bydgoszcz; 2007.

21. Kriege M, Brekelmans C, Obdeijn IM, et al. Factors affecting sensitivity and specificity of screening mammography and MRI in women with an inherited risk for breast cancer. Breast Cancer Res Treat 2006; 100: 109-119.

22. Lohrke J, Frenzel T, Endrikat J, et al. 25 years of contrast-enhanced MRI: developments, current challenges and future perspectives. Adv Ther 2016; 33: 1-28. 